\title{
AN OPTIMAL LIOUVILLE THEOREM FOR THE LINEAR HEAT EQUATION WITH A NONLINEAR BOUNDARY CONDITION
}

\author{
PAVOL QUITTNER \\ Department of Applied Mathematics and Statistics, Comenius University \\ Mlynská dolina, 84248 Bratislava, Slovakia \\ email: quittner@fmph.uniba.sk \\ Dedicated to the memory of Pavol Brunovsky
}

\begin{abstract}
Liouville theorems for scaling invariant nonlinear parabolic problems in the whole space and/or the halfspace (saying that the problem does not posses positive bounded solutions defined for all times $t \in(-\infty, \infty))$ guarantee optimal estimates of solutions of related initial-boundary value problems in general domains. We prove an optimal Liouville theorem for the linear equation in the halfspace complemented by the nonlinear boundary condition $\partial u / \partial \nu=u^{q}, q>1$.
\end{abstract}

Keywords. Liouville theorem, heat equation, nonlinear boundary condition

AMS Classification. 35K60, 35B45, 35B40

\section{InTRODUCTION AND MAIN RESUltS}

Liouville theorems for scaling invariant superlinear parabolic problems in the whole space and/or the halfspace (saying that the problem does not posses positive bounded solutions defined for all times $t \in(-\infty, \infty))$ guarantee optimal estimates of solutions of related initial-boundary value problems in general domains, including estimates of singularities and decay, see [11] or [16] and the references therein. In the case of the model problem

$$
u_{t}-\Delta u=u^{p}, \quad x \in \mathbb{R}^{n}, t \in \mathbb{R},
$$

where $p>1, n \geq 1$ and $u=u(x, t)>0$, an optimal Liouville theorem (i.e. a Liouville theorem valid in the full subcritical range) has been recently proved in [14]. Its proof was inspired by [4] and it was based on refined energy estimates for suitably rescaled solutions. In this paper we adapt the arguments in [14] to prove an optimal Liouville theorem for the problem

$$
\left.\begin{array}{rlrl}
u_{t}-\Delta u & =0 & & \text { in } \mathbb{R}_{+}^{n} \times \mathbb{R}, \\
u_{\nu} & =u^{q} & & \text { on } \partial \mathbb{R}_{+}^{n} \times \mathbb{R},
\end{array}\right\}
$$

Supported in part by the Slovak Research and Development Agency under the contract No. APVV-180308 and by VEGA grant 1/0347/18. 
P. QuitTner

where $u=u(x, t)>0, \mathbb{R}_{+}^{n}:=\left\{\left(x=\left(x_{1}, x_{2}, \ldots, x_{n}\right) \in \mathbb{R}^{n}: x_{1}>0\right\}, \nu=(-1,0,0, \ldots, 0)\right.$ is the outer unit normal on the boundary $\partial \mathbb{R}_{+}^{n}=\left\{x \in \mathbb{R}^{n}: x_{1}=0\right\}$ and $q>1$. In addition, we also provide an application of our Liouville theorem.

The nonexistence of positive classical stationary solutions of (1) is known for $q<q_{S}$, where

$$
q_{S}:= \begin{cases}+\infty & \text { if } n \leq 2 \\ \frac{n}{n-2} & \text { if } n>2\end{cases}
$$

and the condition $q<q_{S}$ is optimal for the nonexistence, see $[7,5]$ and the references therein. Our main result is the following Liouville theorem.

Theorem 1. Let $1<q<q_{S}$. Then problem (1) does not possess positive classical bounded solutions.

The nonexistence result in Theorem 1 follows from the Fujita-type results in $[3,2]$ if $q \leq(n+1) / n$. It has also been proved for $n=1, q>1$ (for solutions with bounded spatial derivatives, see [15]), and for $n \geq 1$ and $q<q_{s g}$ or $q=q_{s g}$ (see [12] or [13], respectively), where

$$
q_{s g}:= \begin{cases}+\infty & \text { if } n \leq 2 \\ \frac{n-1}{n-2} & \text { if } n>2 .\end{cases}
$$

Assuming on the contrary that a solution in Theorem 1 exists, the proof of $[12$, Theorem 5] guarantees that we may assume that the solution is nonincreasing in $x_{1}$. Using this monotonicity, the proof of Theorem 1 could be obtained by more or less straightforward modifications of the proof of [14, Theorem 1]. Unfortunately, several technical arguments in Steps 4-6 of the proof of [14, Theorem 1] are written in an unnecessarily complicated way. In order to make those arguments simpler and more transparent, we have significantly modified the corresponding parts of the proof; see Lemmas 5-8 below. Analogous modifications can also be done in the proof of [14, Theorem 1], see Remark 9 below.

Theorem 1 can be used in order to prove optimal estimates for various problems related to (1). In particular, it guarantees an optimal blow-up rate estimate for positive solutions of the problem

$$
\left.\begin{array}{rlrl}
u_{t}-\Delta u & =0 & & x \in \Omega, t \in(0, T), \\
u_{\nu} & =u^{q} & & x \in \partial \Omega, t \in(0, T),
\end{array}\right\}
$$

where $\Omega \subset \mathbb{R}^{n}$ is bounded and smooth. More precisely, the proof of [12, Theorem 7] shows that the following theorem is true (see the discussion after [12, Theorem 7] for related results and references on the blow-up rate and see also [6] and the references therein for results on the blow-up profile).

Theorem 2. Assume that $\Omega \subset \mathbb{R}^{n}$ is bounded and smooth, $1<q<q_{S}$. Assume also that $u$ is a positive classical solution of (2) which blows up at $t=T$. Then there exists $C=C(u)>0$ such that $u$ satisfies the blow-up rate estimate

$$
u(x, t)(T-t)^{1 / 2(q-1)}+|\nabla u(x, t)|(T-t)^{q / 2(q-1)} \leq C
$$

for all $x \in \bar{\Omega}$ and $t \in(T / 2, T)$. 


\section{Proof of Theorem 1}

The proof is a combination of arguments used in the proofs of [12, Theorem 5] and [14, Theorem 1].

Assume on the contrary that there exists a positive bounded solution $u$ of (1). As in the proof of [12, Theorem 5] we may assume

$$
u(x, t)+|\nabla u(x, t)| \leq 1 \quad \text { for all } x \in \overline{\mathbb{R}_{+}^{n}}, t \in \mathbb{R},
$$

and we also have

$$
u_{x_{1}}(x, t) \leq 0 \quad \text { for all } x \in \mathbb{R}_{+}^{n}, t \in \mathbb{R} .
$$

Due to the results in $[12,13]$ we may also assume $q>q_{s g}$. We set $\beta:=\frac{1}{2(q-1)}$ and by $C, C_{0}, C_{1}, \ldots, c, c_{0}, c_{1}, \ldots$ we will denote positive constants which depend only on $n$ and $q$; the constants $C, c$ may vary from step to step. Finally, $M=M(n, q)$ will denote a positive integer (the number of bootstrap steps). The proof will be divided into several steps.

Step 1: Initial estimates. For $y \in \mathbb{R}_{+}^{n}, s \in \mathbb{R}, a \in \partial \mathbb{R}_{+}^{n}$ and $k=1,2, \ldots$ set

$$
w(y, s)=w_{k}^{a}(y, s):=(k-t)^{\beta} u(y \sqrt{k-t}+a, t), \quad \text { where } s=-\log (k-t), \quad t<k .
$$

Set also $s_{k}:=-\log k$ and notice that $w=w_{k}^{a}$ solve the problem

$$
\left.\begin{array}{ll}
w_{s}=\Delta w-\frac{1}{2} y \cdot \nabla w-\beta w=\frac{1}{\rho} \nabla \cdot(\rho \nabla w)-\beta w & \text { in } \mathbb{R}_{+}^{n} \times \mathbb{R}, \\
w_{\nu}=w^{q} & \text { on } \partial \mathbb{R}_{+}^{n} \times \mathbb{R},
\end{array}\right\}
$$

where $\rho(y):=e^{-|y|^{2} / 4}$. In addition, $w_{k}^{a}\left(0, s_{k}\right)=k^{\beta} u(a, 0)$ and

$$
\left\|w_{k}^{a}(\cdot, s)\right\|_{\infty} \leq C_{0} k^{\beta}\|u(\cdot, t)\|_{\infty} \leq C_{0} k^{\beta} \quad \text { for } s \in\left[s_{k}-M-1, \infty\right),
$$

where $t=k-e^{-s}$ and $C_{0}:=e^{(M+1) \beta}$. Set

$E(s)=E_{k}^{a}(s):=\frac{1}{2} \int_{\mathbb{R}_{+}^{n}}\left(\left|\nabla w_{k}^{a}\right|^{2}+\beta\left(w_{k}^{a}\right)^{2}\right)(y, s) \rho(y) d y-\frac{1}{q+1} \int_{\partial \mathbb{R}_{+}^{n}}\left(w_{k}^{a}\right)^{q+1}(y, s) \rho(y) d S_{y}$.

Multiplying equation (4) by $w \rho$ and integrating over $y \in \mathbb{R}_{+}^{n}$ we obtain

$$
E(s)=-\frac{1}{2} \int_{\mathbb{R}_{+}^{n}}\left(w w_{s}\right)(y, s) \rho(y) d y+\frac{1}{2} \frac{q-1}{q+1} \int_{\partial \mathbb{R}_{+}^{n}} w^{q+1}(y, s) \rho(y) d S_{y} .
$$

The function $s \mapsto E(s)$ is nonincreasing and nonnegative (see [1]). Integrating (6) over the time interval $\left(\sigma_{1}, \sigma_{2}\right)$ we have

$$
\left.\begin{array}{rl}
\frac{1}{2}\left(\int_{\mathbb{R}_{+}^{n}} w^{2}\left(y, \sigma_{2}\right) \rho(y) d y-\int_{\mathbb{R}_{+}^{n}} w^{2}\left(y, \sigma_{1}\right) \rho(y) d y\right) & \\
=-2 \int_{\sigma_{1}}^{\sigma_{2}} E(s) d s+\frac{q-1}{q+1} \int_{\sigma_{1}}^{\sigma_{2}} \int_{\partial \mathbb{R}_{+}^{n}} w^{q+1}(y, s) \rho(y) d S_{y} d s .
\end{array}\right\}
$$

As in the proof of [12, Theorem 5] we also obtain

$$
\int_{\sigma_{1}}^{\sigma_{2}} \int_{\mathbb{R}_{+}^{n}}\left|\frac{\partial w}{\partial s}(y, s)\right|^{2} \rho(y) d y d s=E\left(\sigma_{1}\right)-E\left(\sigma_{2}\right) \leq E\left(\sigma_{1}\right),
$$




$$
\begin{gathered}
\int_{\mathbb{R}_{+}^{n}} w^{r}(y, s) \rho(y) d y \leq C \int_{\partial \mathbb{R}_{+}^{n}} w^{r}(y, s) \rho(y) d S_{y}, \quad r \geq 1, \\
\int_{\mathbb{R}_{+}^{n}} w(y, s) \rho(y) d y \leq C, \\
\int_{\sigma_{1}}^{\sigma_{2}} \int_{\partial \mathbb{R}_{+}^{n}} w^{q}(y, s) \rho(y) d S_{y} d s \leq C\left(1+\sigma_{2}-\sigma_{1}\right) .
\end{gathered}
$$

Given $1 \leq m \leq M$, the monotonicity of $E,(7),(5)$, (10) and (11) guarantee

$$
\begin{aligned}
& 2 E_{k}^{a}\left(s_{k}-m\right) \leq 2 \int_{s_{k}-m-1}^{s_{k}-m} E_{k}^{a}(s) d s \\
& \quad \leq \frac{1}{2} \int_{\mathbb{R}_{+}^{n}}\left(w_{k}^{a}\right)^{2}\left(y, s_{k}-m-1\right) \rho(y) d y+\frac{q-1}{q+1} \int_{s_{k}-m-1}^{s_{k}-m} \int_{\partial \mathbb{R}_{+}^{n}}\left(w_{k}^{a}\right)^{q+1}(y, s) \rho(y) d S_{y} d s \\
& \quad \leq C k^{\beta}\left(\int_{\mathbb{R}_{+}^{n}} w_{k}^{a}\left(y, s_{k}-m-1\right) \rho(y) d y+\int_{s_{k}-m-1}^{s_{k}-m} \int_{\partial \mathbb{R}_{+}^{n}}\left(w_{k}^{a}\right)^{q}(y, s) \rho(y) d S_{y} d s\right) \\
& \quad \leq C k^{\beta} .
\end{aligned}
$$

Consequently,

$$
E_{k}^{a}\left(s_{k}-M\right) \leq C k^{\beta}
$$

Notice also that (8) guarantees

$$
\int_{s_{k}-m}^{s_{k}-m+1} \int_{\mathbb{R}_{+}^{n}}\left|\frac{\partial w_{k}^{a}}{\partial s}(y, s)\right|^{2} \rho(y) d y d s \leq E_{k}^{a}\left(s_{k}-m\right), \quad m=1,2, \ldots M
$$

Step 2: The plan of the proof. We will show that there exist an integer $M=M(n, q)$ and positive numbers $\gamma_{m}, m=1,2, \ldots M$, such that

$$
\gamma_{1}<\gamma_{2}<\cdots<\gamma_{M}=\beta, \quad \gamma_{1}<\mu:=2 \beta-\frac{n-2}{2},
$$

and

$$
E_{k}^{a}\left(s_{k}-m\right) \leq C k^{\gamma_{m}}, \quad a \in \partial \mathbb{R}_{+}^{n}, k \text { large, }
$$

where $m=M, M-1, \ldots, 1$, and " $k$ large" means $k \geq k_{0}$ with $k_{0}=k_{0}(n, q, u)$. Then, taking $\lambda_{k}:=k^{-1 / 2}$ and setting

$$
v_{k}(z, \tau):=\lambda_{k}^{1 /(q-1)} w_{k}^{0}\left(\lambda_{k} z, \lambda_{k}^{2} \tau+s_{k}\right), \quad z \in \mathbb{R}_{+}^{n},-k \leq \tau \leq 0,
$$

we obtain $0<v_{k} \leq C, v_{k}(0,0)=u(0,0)$,

$$
\begin{aligned}
\frac{\partial v_{k}}{\partial \tau}-\Delta v_{k} & =-\lambda_{k}^{2}\left(\frac{1}{2} z \cdot \nabla v_{k}+\beta v_{k}\right) & & \text { in } \mathbb{R}_{+}^{n} \times(-k, 0), \\
\left(v_{k}\right)_{\nu} & =v_{k}^{q} & & \text { on } \partial \mathbb{R}_{+}^{n} \times(-k, 0) .
\end{aligned}
$$

In addition, using (13) and (14) with $m=1$ we also have

$$
\begin{aligned}
\int_{-k}^{0} \int_{|z|<\sqrt{k}, z_{1}>0}\left|\frac{\partial v_{k}}{\partial \tau}(z, \tau)\right|^{2} d z d \tau & =\lambda_{k}^{2 \mu} \int_{s_{k}-1}^{s_{k}} \int_{|y|<1, y_{1}>0}\left|\frac{\partial w_{k}^{0}}{\partial s}(y, s)\right|^{2} d y d s \\
& \leq C k^{-\mu+\gamma_{1}} \rightarrow 0 \text { as } k \rightarrow \infty .
\end{aligned}
$$


Now a priori estimates of $v_{k}$ (see estimates in [9, Theorem 7.2 and the subsequent Remark] or [10, Theorem 13.16] applied to $v_{k}$ and their first order derivatives, and cf. also [8, (3.9)], for example) show that (up to a subsequence) the sequence $\left\{v_{k}\right\}$ converges to a positive solution $v=v(z)$ of the problem $\Delta v=0$ in $\mathbb{R}_{+}^{n}, v_{\nu}=v^{q}$ on $\partial \mathbb{R}_{+}^{n}$ which contradicts the elliptic Liouville theorem in [7]. This contradiction will conclude the proof.

Notice that (14) is true if $m=M$ due to (12). In the rest of the proof we consider $M>1$, fix $m \in\{M, M-1, \ldots, 2\}$, assume that (14) is true with this fixed $m$, and we will prove that (14) remains true with $m$ replaced by $m-1$. More precisely, we assume

$$
E_{k}^{a}\left(s_{k}-m\right) \leq C k^{\gamma}, \quad a \in \partial \mathbb{R}_{+}^{n}, k \text { large, }
$$

(where $\gamma:=\gamma_{m} \in[\mu, \beta]$ ) and we will show that

$$
E_{k}^{a}\left(s_{k}-m+1\right) \leq C k^{\tilde{\gamma}}, \quad a \in \partial \mathbb{R}_{+}^{n}, k \text { large, }
$$

where $\tilde{\gamma}<\gamma$ (and then we set $\gamma_{m-1}:=\tilde{\gamma}$ ). Our proof shows that there exists an open neighbourhood $U=U(n, q, \gamma)$ of $\gamma$ such that (17) remains true also if (16) is satisfied with $\gamma$ replaced by any $\gamma^{\prime} \in U$. The compactness of $[\mu, \beta]$ guarantees that the difference $\gamma-\tilde{\gamma}$ can be bounded below by a positive constant $\delta=\delta(n, q)$ for all $\gamma \in[\mu, \beta]$, hence there exists $M=M(n, q)$ such that $\gamma_{1}<\mu \leq \gamma_{2}$.

Step 3: Notation and auxiliary results. In the rest of the proof we will also use the following notation and facts: If $Z$ is a finite set or a measurable subset of $\mathbb{R}^{d}$, then by $\# Z$ or $|Z|$ we denote the cardinality or the $d$-dimensional measure of $Z$, respectively. Set

$$
\begin{aligned}
& C(M):=8 n e^{M+1}, \quad B_{r}^{\partial}(a):=\left\{x \in \partial \mathbb{R}_{+}^{n}:|x-a| \leq r\right\}, \quad B_{r}^{\partial}:=B_{r}^{\partial}(0), \\
& R_{k}:=\sqrt{8 n \log k}, \quad B_{r}^{+}(a):=\left\{x \in \mathbb{R}_{+}^{n}:|x-a| \leq r\right\}, \quad B_{r}^{+}:=B_{r}^{+}(0) .
\end{aligned}
$$

Given $a \in \partial \mathbb{R}_{+}^{n}$, there exists an integer $X=X(n, k)$ and there exist $a^{1}, a^{2}, \ldots a^{X} \in \partial \mathbb{R}_{+}^{n}$ (depending on $a, n, k)$ such that $a^{1}=a, X \leq C(\log k)^{(n-1) / 2}$ and

$$
D^{k}(a):=B_{\sqrt{C(M) k \log (k)}}^{\partial}(a) \subset \bigcup_{i=1}^{X} B_{\sqrt{k} / 2}^{\partial}\left(a^{i}\right) .
$$

Notice that if $y \in B_{R_{k}}^{\partial}$ and $s \in\left[s_{k}-M-1, s_{k}\right]$, then $a+y e^{-s / 2} \in D^{k}(a)$, hence (18) guarantees the existence of $i \in\{1,2, \ldots, X\}$ such that

$$
w_{k}^{a}(y, s)=w_{k}^{a^{i}}\left(y^{i}, s\right), \quad \text { where } \quad y^{i}:=y+\left(a-a^{i}\right) e^{s / 2} \in B_{1 / 2}^{\partial} .
$$

The contradiction argument in Step 2 based on the nonexistence of positive stationary solutions of (1), combined with a doubling argument can be used to obtain the following useful pointwise estimates of the solution $u$.

Lemma 3. Let $M, s_{k}, w_{k}^{a}$ be as above, $\zeta \in \mathbb{R}, \xi, C^{*}>0, d_{k}, r_{k} \in(0,1], k=1,2, \ldots$ Set

$$
\begin{aligned}
\mathcal{T}_{k} & =\mathcal{T}_{k}\left(d_{k}, r_{k}, \zeta, C^{*}\right) \\
& :=\left\{(a, \sigma, b) \in \partial \mathbb{R}_{+}^{n} \times\left(s_{k}-M, s_{k}\right] \times \partial \mathbb{R}_{+}^{n}: \int_{\sigma-d_{k}}^{\sigma} \int_{B_{r_{k}}^{+}(b)}\left(w_{k}^{a}\right)_{s}^{2} d y d s \leq C^{*} k^{\zeta}\right\} .
\end{aligned}
$$

Assume

$$
\xi \frac{\mu}{\beta}>\zeta \quad \text { and } \quad \frac{1}{\log (k)} \min \left(d_{k} k^{\xi / \beta}, r_{k} k^{\xi / 2 \beta}\right) \rightarrow \infty \quad \text { as } \quad k \rightarrow \infty
$$


Then there exists $k_{1}$ such that

$$
w_{k}^{a}(y, \sigma) \leq k^{\xi} \quad \text { whenever } \quad y \in B_{r_{k} / 2}^{\partial}(b), \quad k \geq k_{1} \quad \text { and }(a, \sigma, b) \in \mathcal{T}_{k} .
$$

Proof. Assume on the contrary that there exist $k_{1}, k_{2} \ldots$ with the following properties: $k_{j} \rightarrow \infty$ as $j \rightarrow \infty$, and for each $k \in\left\{k_{1}, k_{2}, \ldots\right\}$ there exist $\left(a_{k}, \sigma_{k}, b_{k}\right) \in \mathcal{T}_{k}$ and $y_{k} \in B_{r_{k} / 2}^{\partial}\left(b_{k}\right)$ such that $\tilde{w}_{k}\left(y_{k}, \sigma_{k}\right)>k^{\xi}$, where $\tilde{w}_{k}:=w_{k}^{a_{k}}$.

Given $k \in\left\{k_{1}, k_{2} \ldots\right\}$, we can choose an integer $K$ such that

$$
2^{K} k^{\xi}>C_{0} k^{\beta}, \quad K<C \log k .
$$

Set

$$
Z_{j}:=B_{r_{k}(1 / 2+j /(2 K))}^{\partial}\left(b_{k}\right) \times\left[\sigma_{k}-d_{k}(1 / 2+j /(2 K)), \sigma_{k}\right], \quad j=0,1, \ldots, K .
$$

Then

$$
B_{r_{k} / 2}^{\partial}\left(b_{k}\right) \times\left[\sigma_{k}-d_{k} / 2, \sigma_{k}\right]=Z_{0} \subset Z_{1} \subset \cdots \subset Z_{K}=B_{r_{k}}^{\partial}\left(b_{k}\right) \times\left[\sigma_{k}-d_{k}, \sigma_{k}\right] .
$$

Since $\sup _{Z_{0}} \tilde{w}_{k} \geq \tilde{w}_{k}\left(y_{k}, \sigma_{k}\right)>k^{\xi}$, estimates (21) and (5) imply the existence of $j^{*} \in$ $\{0,1, \ldots K-1\}$ such that

$$
2 \sup _{Z_{j^{*}}} \tilde{w}_{k} \geq \sup _{Z_{j^{*}+1}} \tilde{w}_{k}
$$

(otherwise $C_{0} k^{\beta} \geq \sup _{Z_{K}} \tilde{w}_{k}>2^{K} \sup _{Z_{0}} \tilde{w}_{k}>2^{K} k^{\xi}$, a contradiction). Fix $\left(\hat{y}_{k}, \hat{s}_{k}\right) \in Z_{j^{*}}$ such that

$$
W_{k}:=\tilde{w}_{k}\left(\hat{y}_{k}, \hat{s}_{k}\right)=\sup _{Z_{j^{*}}} \tilde{w}_{k} .
$$

Then $W_{k} \geq k^{\xi}, B_{r_{k} /(2 K)}^{\partial}\left(\hat{y}_{k}\right) \times\left[\hat{s}_{k}-\frac{d_{k}}{2 K}, \hat{s}_{k}\right] \subset Z_{j^{*}+1}$, hence (3) implies

$$
\tilde{w}_{k} \leq 2 W_{k} \quad \text { on } \quad \hat{Q}_{k}:=B_{r_{k} /(2 K)}^{+}\left(\hat{y}_{k}\right) \times\left[\hat{s}_{k}-\frac{d_{k}}{2 K}, \hat{s}_{k}\right] .
$$

Set $\lambda_{k}:=W_{k}^{-1 /(2 \beta)}$ (hence $\lambda_{k} \leq k^{-\xi /(2 \beta)} \rightarrow 0$ as $k \rightarrow \infty$ ) and

$$
v_{k}(z, \tau):=\lambda_{k}^{2 \beta} \tilde{w}_{k}\left(\lambda_{k} z+\hat{y}_{k}, \lambda_{k}^{2} \tau+\hat{s}_{k}\right) .
$$

Then $v_{k}(0,0)=1, v_{k} \leq 2$ on $Q_{k}:=B_{r_{k} /\left(2 K \lambda_{k}\right)}^{+} \times\left[-d_{k} /\left(2 K \lambda_{k}^{2}\right), 0\right]$, and

$$
\begin{aligned}
\frac{\partial v_{k}}{\partial \tau}-\Delta v_{k} & =-\lambda_{k}^{2}\left(\frac{1}{2} z \cdot \nabla v_{k}+\beta v_{k}\right) & & \text { in } Q_{k}, \\
\left(v_{k}\right)_{\nu} & =v_{k}^{q} & & \text { on } Q_{k}^{\partial},
\end{aligned}
$$

where $Q_{k}^{\partial}:=B_{r_{k} /\left(2 K \lambda_{k}\right)}^{\partial} \times\left[-d_{k} /\left(2 K \lambda_{k}^{2}\right), 0\right]$. In addition, as $k \rightarrow \infty$,

$$
\frac{r_{k}}{2 K \lambda_{k}} \geq \frac{r_{k} k^{\xi /(2 \beta)}}{C \log (k)} \rightarrow \infty, \quad \frac{d_{k}}{2 K \lambda_{k}^{2}} \geq \frac{d_{k} k^{\xi / \beta}}{C \log (k)} \rightarrow \infty .
$$

Since $\left(a_{k}, \sigma_{k}, b_{k}\right) \in \mathcal{T}_{k}$ and $\hat{Q}_{k} \subset B_{r_{k}}^{+}\left(b_{k}\right) \times\left[\sigma_{k}-d_{k}, \sigma_{k}\right]$, we obtain

$$
\int_{Q_{k}}\left|\frac{\partial v_{k}}{\partial \tau}(z, \tau)\right|^{2} d z d \tau=\lambda_{k}^{2 \mu} \int_{\hat{Q}_{k}}\left|\frac{\partial \tilde{w}_{k}}{\partial s}(y, s)\right|^{2} d y d s \leq C^{*} k^{\delta}, \quad \text { where } \delta:=-\xi \frac{\mu}{\beta}+\zeta<0 \text {. }
$$


Hence, as above, a suitable subsequence of $\left\{v_{k}\right\}$ converges to a positive solution $v=v(z)$ of the problem $\Delta v=0$ in $\mathbb{R}_{+}^{n}, v_{\nu}=v^{q}$ on $\partial \mathbb{R}_{+}^{n}$, which contradicts the elliptic Liouville theorem in [7].

Remark 4. By a simple modification of the proof of Lemma 3 one can show that the estimate $w_{k}^{a}(y, \sigma) \leq k^{\xi}$ can be improved to

$$
w_{k}^{a}(y, \sigma)+\left|\nabla w_{k}^{a}(y, \sigma)\right|^{1 / q}+\left|\left(w_{k}^{a}\right)_{s}(y, \sigma)\right|^{1 /(2 q-1)} \leq k^{\xi} .
$$

In fact, set $\tilde{w}_{k}:=w_{k}^{a_{k}}+\left|\nabla w_{k}^{a_{k}}\right|^{1 / q}+\left|\left(w_{k}^{a_{k}}\right)_{s}\right|^{1 /(2 q-1)}$ and assume on the contrary that $\tilde{w}_{k}\left(y_{k}, \sigma_{k}\right)>k^{\xi}$, where $y_{k}, \sigma_{k}, k$ are as in the proof of Lemma 3. Repeat the doubling estimates (with a modified constant $C_{0}$ ) and define $W_{k}$ and $\lambda_{k}$ as in that proof, but replace $\tilde{w}_{k}$ with $w_{k}^{a_{k}}$ in the definition of $v_{k}$. Then $v_{k}$ solves $(22)$,

$$
\begin{aligned}
\left(v_{k}+\left|\nabla v_{k}\right|^{1 / q}+\left|\left(v_{k}\right)_{\tau}\right|^{1 /(2 q-1)}\right)(0,0) & =1 \\
v_{k}+\left|\nabla v_{k}\right|^{1 / q}+\left|\left(v_{k}\right)_{\tau}\right|^{1 /(2 q-1)} & \leq 2 \text { in } Q_{k},
\end{aligned}
$$

and passing to the limit we arrive at a contradiction.

Recall that $\gamma \in[\mu, \beta]$ (see $(16))$.

Lemma 5. Let $\mathcal{T}_{k}=\mathcal{T}_{k}\left(d_{k}, r_{k}, \zeta, C^{*}\right)$ be as in Lemma 3, $\omega \in \mathbb{R}, \varepsilon, C_{1}>0$,

$$
0 \leq \alpha<\frac{\xi}{\beta}, \quad \xi \frac{\mu}{\beta}>\gamma-\alpha+\varepsilon-\omega,
$$

and assume

$$
(a, \sigma, 0) \in \mathcal{T}_{k}\left(\frac{1}{2} k^{-\alpha}, 1, \gamma-\alpha+\varepsilon, C_{1}\right) \text { for } k \text { large }
$$

Set

Then

$$
G:=\left\{y \in B_{1 / 2}^{\partial}: w_{k}^{a}(y, \sigma) \leq k^{\xi}\right\}
$$

$$
\left|B_{1 / 2}^{\partial} \backslash G\right| \leq C k^{\omega-(n-1) \alpha / 2} \text { for } k \text { large. }
$$

Proof. There exist $b^{1}, \ldots, b^{Y} \in \partial \mathbb{R}_{+}^{n}$ with $Y \leq C k^{(n-1) \alpha / 2}$ such that

$$
B_{1 / 2}^{\partial} \subset \bigcup_{j=1}^{Y} B^{j}, \quad \text { where } \quad B^{j}:=B_{\frac{1}{2} k^{-\alpha / 2}}^{\partial}\left(b^{j}\right),
$$

and

$$
\#\left\{j: y \in B_{k^{-\alpha / 2}}^{+}\left(b^{j}\right)\right\} \leq C_{n} \quad \text { for any } y \in \mathbb{R}_{+}^{n} .
$$

Set

$$
\begin{aligned}
H & :=\left\{j \in\{1,2, \ldots, Y\}:\left(a, \sigma, b^{j}\right) \in \mathcal{T}_{k}\left(\frac{1}{2} k^{-\alpha}, k^{-\alpha / 2}, \gamma-\alpha+\varepsilon-\omega, C_{1} C_{n}\right)\right\}, \\
H^{c} & :=\{1,2, \ldots, Y\} \backslash H .
\end{aligned}
$$

If $j \in H$, then Lemma 3 guarantees $w_{k}^{a}(y, \sigma) \leq k^{\xi}$ for $y \in B^{j}$. Consequently,

$$
B_{1 / 2}^{\partial} \cap \bigcup_{j \in H} B^{j} \subset G, \text { hence } B_{1 / 2}^{\partial} \backslash G \subset \bigcup_{j \in H^{c}} B^{j} .
$$

Now (23), the definition of $H$ and (25) imply $\# H^{c}<k^{\omega}$, hence (24) is true. 
Lemma 6. Fix a positive integer $L=L(n, q)$ such that

$$
\beta\left(\frac{q+1}{3 q-1}\right)^{L}<\mu .
$$

If $\varepsilon, \delta>0$ are small enough, then there exist $\xi_{\ell}, \alpha_{\ell}, \omega_{\ell}, \ell=1,2, \ldots L$, such that

$$
\gamma-\delta-\varepsilon>\xi_{1} \leq \xi_{2} \leq \cdots \leq \xi_{L} \leq \beta=: \xi_{L+1}
$$

and the following inequalities are true for $\ell=1,2, \ldots, L$ :

$$
0 \leq \alpha_{\ell}<\frac{\xi_{\ell}}{\beta}, \quad \xi_{l} \frac{\mu}{\beta}>\gamma-\alpha_{\ell}+\varepsilon-\omega_{\ell}, \quad \omega_{\ell}-\frac{(n-1) \alpha_{\ell}}{2} \leq \gamma-\delta-(q+1) \xi_{\ell+1} .
$$

Proof. Consider $\xi \in[\mu / 2, \beta]$ and $\tilde{\xi} \in\left[\xi, \frac{3 q-1}{q+1} \xi\right)$. Set also $\alpha:=\frac{\xi}{\beta}-\varepsilon_{\alpha}$, where $\varepsilon_{\alpha}>0$ is small. Since

$$
\gamma-\frac{\xi}{\beta}-\xi \frac{\mu}{\beta}=\frac{n-1}{2} \frac{\xi}{\beta}+\gamma-(3 q-1) \xi
$$

and $(q+1) \tilde{\xi}<(3 q-1) \xi$, we see that

$$
\underline{\omega}:=\gamma-\alpha+\varepsilon-\xi \frac{\mu}{\beta}<\frac{n-1}{2} \alpha+\gamma-\delta-(q+1) \tilde{\xi}=: \bar{\omega}
$$

provided $\varepsilon, \varepsilon_{\alpha}, \delta$ are small enough. Consequently, we may choose $\omega \in(\underline{\omega}, \bar{\omega})$.

If $\varepsilon, \delta$ are small enough, then (26) guarantees the existence of $\xi_{1}, \ldots, \xi_{L}$ satisfying (27), $\xi_{1} \geq \mu / 2$ and $\xi_{\ell+1}<\frac{3 q-1}{q+1} \xi_{\ell}$ for $\ell=1,2, \ldots, L$. Fix $\ell \in\{1,2, \ldots, L\}$, set $\xi:=\xi_{\ell}, \tilde{\xi}:=\xi_{\ell+1}$, and let $\alpha, \omega$ be as above. Set $\alpha_{\ell}:=\alpha, \omega_{\ell}:=\omega$. Then the definitions of $\alpha_{\ell}, \omega_{\ell}$ and (29) guarantee $(28)$.

Lemma 7. Let $L, \varepsilon, \delta$ and $\xi_{\ell}, \alpha_{\ell}, \omega_{\ell}, \ell=1,2, \ldots L$, be as in Lemma 6 and let $\mathcal{T}_{k}=$ $\mathcal{T}_{k}\left(d_{k}, r_{k}, \zeta, C^{*}\right)$ be as in Lemma 3. Assume $(a, \sigma, 0) \in \mathcal{T}_{k}\left(\frac{1}{2} k^{-\alpha_{\ell}}, 1, \gamma-\alpha_{\ell}+\varepsilon, C\right)$ for $\ell=1,2, \ldots L$ and $k$ large and

$$
\int_{B_{1 / 2}^{\partial}}\left(w_{k}^{a}\right)^{q}(y, \sigma) d S_{y} \leq C k^{\varepsilon} \text { for } k \text { large. }
$$

Then

$$
\int_{B_{1 / 2}^{\partial}}\left(w_{k}^{a}\right)^{q+1}(y, \sigma) d S_{y} \leq C k^{\gamma-\delta} \text { for } k \text { large. }
$$

Proof. Given $\ell \in\{1,2, \ldots, L\}$, set $\xi=\xi_{\ell}, \alpha=\alpha_{\ell}, \omega=\omega_{\ell}$, and let $G$ be the set in Lemma 5 . Set $G_{\ell}:=G$ and $G_{L+1}:=B_{1 / 2}^{\partial}$. Lemma 5 and (28) guarantee

$$
\left|G_{\ell+1} \backslash G_{\ell}\right| \leq\left|B_{1 / 2}^{\partial} \backslash G_{\ell}\right| \leq C k^{\omega_{\ell}-(n-1) \alpha_{\ell} / 2} \leq C k^{\gamma-\delta-(q+1) \xi_{\ell+1}}
$$

hence

$$
\int_{G_{\ell+1} \backslash G_{\ell}}\left(w_{k}^{a}\right)^{q+1}(y, \sigma) d S_{y} \leq C k^{(q+1) \xi_{\ell+1}}\left|G_{\ell+1} \backslash G_{\ell}\right| \leq C k^{\gamma-\delta} .
$$

In addition, the definition of $G_{1},(30)$ and (27) imply

$$
\int_{G_{1}}\left(w_{k}^{a}\right)^{q+1}(y, \sigma) d S_{y} \leq k^{\xi_{1}} \int_{G_{1}}\left(w_{k}^{a}\right)^{q}(y, \sigma) d S_{y} \leq C k^{\xi_{1}+\varepsilon} \leq C k^{\gamma-\delta} .
$$

Since $B_{1 / 2}^{\partial}=G_{1} \cup \bigcup_{\ell=1}^{L}\left(G_{\ell+1} \backslash G_{\ell}\right)$, the conclusion follows. 
Step 4: The choice of a suitable time. The proof of (17) will be based on estimates of $w_{k}^{a^{i}}\left(\cdot, s^{*}\right), i=1,2, \ldots, X$, where $s^{*}=s^{*}(k, a) \in\left[s_{k}-m, s_{k}-m+1\right]$ is a suitable time.

Lemma 8. Let $\varepsilon, \gamma, C_{1}, C_{2}>0, \alpha_{1}, \alpha_{2}, \ldots, \alpha_{L} \geq 0$, and, given $k=1,2, \ldots$, let $X_{k}$ be a positive integer satisfying $X_{k} \leq k^{\varepsilon / 2}$ and $\sigma_{k} \in \mathbb{R}$. Set $J_{k}:=\left[\sigma_{k}, \sigma_{k}+1\right]$, $\tilde{J}_{k}:=$ $\left[\sigma_{k}+1 / 2, \sigma_{k}+1\right]$, and assume that $f_{k}^{1}, \ldots, f_{k}^{X_{k}}, g_{k}^{1}, \ldots, g_{k}^{X_{k}} \in C\left(J_{k}, \mathbb{R}^{+}\right)$satisfy

$$
\int_{J_{k}} f_{k}^{i}(s) d s \leq C_{1} k^{\gamma}, \quad \int_{J_{k}} g_{k}^{i}(s) d s \leq C_{2}, \quad i=1,2, \ldots X_{k}, k=1,2, \ldots
$$

Then there exists $k_{1}=k_{1}(\varepsilon, L)$ with the following property: If $k \geq k_{1}$, then there exists $s^{*}=s^{*}(k) \in \tilde{J}_{k}$ such that

$$
\int_{s^{*}-\frac{1}{2} k^{-\alpha_{\ell}}}^{s^{*}} f_{k}^{i}(s) d s \leq C_{1} k^{\gamma-\alpha_{\ell}+\varepsilon}, \quad f_{k}^{i}\left(s^{*}\right) \leq C_{1} k^{\gamma+\varepsilon}, \quad g_{k}^{i}\left(s^{*}\right) \leq C_{2} k^{\varepsilon}
$$

for all $i=1,2, \ldots, X_{k}$ and $\ell=1,2, \ldots, L$.

Proof. Set

$$
h_{k}^{i, \ell}(s):=\int_{s-\frac{1}{2} k^{-\alpha_{\ell}}}^{s} f_{k}^{i}(\tau) d \tau, \quad s \in \tilde{J}_{k}, \quad i=1,2, \ldots X_{k}, \quad \ell=1,2, \ldots, L, \quad k=1,2, \ldots
$$

Then

$$
\begin{gathered}
\int_{\tilde{J}_{k}} h_{k}^{i, \ell}(s) d s=\int_{\tilde{J}_{k}} \int_{s-\frac{1}{2} k^{-\alpha_{\ell}}}^{s} f_{k}^{i}(\tau) d \tau d s=\int_{\tilde{J}_{k}} \int_{0}^{\frac{1}{2} k^{-\alpha_{\ell}}} f_{k}^{i}(s-\tau) d \tau d s \\
=\int_{0}^{\frac{1}{2} k^{-\alpha_{\ell}}} \int_{\tilde{J}_{k}} f_{k}^{i}(s-\tau) d s d \tau \leq \int_{0}^{\frac{1}{2} k^{-\alpha_{\ell}}} \int_{J_{k}} f_{k}^{i}(s) d s d \tau \leq C_{1} k^{\gamma-\alpha_{\ell}}
\end{gathered}
$$

Set

$$
\begin{aligned}
A_{k}^{i} & :=\left\{s \in \tilde{J}_{k}: f_{k}^{i}(s)>C_{1} k^{\gamma+\varepsilon}\right\}, \quad B_{k}^{i}:=\left\{s \in \tilde{J}_{k}: g_{k}^{i}(s)>C_{2} k^{\varepsilon}\right\}, \\
C_{k}^{i, \ell} & :=\left\{s \in \tilde{J}_{k}: h_{k}^{i, \ell}(s)>C_{1} k^{\gamma-\alpha_{\ell}+\varepsilon}\right\} .
\end{aligned}
$$

Then (33) and (34) show that the measure of each of the sets $A_{k}^{i}, B_{k}^{i}, C_{k}^{i, \ell}$ can be estimated above by $k^{-\varepsilon}$. Since the number of these sets (with given index $k$ ) is $(L+2) X_{k} \leq$ $(L+2) k^{\varepsilon / 2}$, their union $U_{k}:=\bigcup_{i} A_{k}^{i} \cup \bigcup_{i} B_{k}^{i} \cup \bigcup_{i, \ell} C_{k}^{i, \ell}$ has measure less than $1 / 2$ for $k \geq k_{1}$, hence for $k \geq k_{1}$ there exists $s^{*}=s^{*}(k) \in \tilde{J}_{k} \backslash U_{k}$. Obviously, $s^{*}$ has the required properties.

Consider $m, \gamma \in[\mu, \beta]$ and $a \in \partial \mathbb{R}_{+}^{n}$ fixed, $J_{k}:=\left[s_{k}-m, s_{k}-m+1\right]$, and let $a^{i}$, $i=1,2, \ldots, X$ be as in (18) (recall that $a^{i}$ and $X$ depend on $k ; X \leq C(\log k)^{(n-1) / 2}$ ). Let $L, \varepsilon$ and $\alpha_{\ell}, \ell=1,2, \ldots, L$ be from Lemma 6 . Set

$$
f_{k}^{i}(s):=\int_{\mathbb{R}_{+}^{n}}\left(w_{k}^{a^{i}}\right)_{s}^{2}(y, s) \rho(y) d y, \quad g_{k}^{i}(s):=\int_{\partial \mathbb{R}_{+}^{n}}\left(w_{k}^{a^{i}}\right)^{q}(y, s) \rho(y) d S_{y}, \quad i=1,2, \ldots X .
$$

Estimates (13), (16) and (11) guarantee that the assumptions of Lemma 8 are satisfied with $C_{1}, C_{2}$ independent of $a$. Consequently, if $k \geq k_{1}$, then there exists $s^{*}=s^{*}(k, a) \in$ 
$\tilde{J}_{k}:=\left[s_{k}-m+1 / 2, s_{k}-m+1\right]$ such that the following estimates are true for $a \in \partial \mathbb{R}^{n}$, $w=w_{k}^{a^{i}}, i=1,2, \ldots X, \ell=1,2, \ldots L$ :

$$
\left.\begin{array}{c}
\int_{s^{*}-\frac{1}{2} k^{-\alpha_{\ell}}}^{s^{*}} \int_{\mathbb{R}_{+}^{n}} w_{s}^{2} \rho d y d s \leq C_{1} k^{\gamma-\alpha_{\ell}+\varepsilon}, \\
\int_{\mathbb{R}_{+}^{n}} w_{s}^{2}\left(y, s^{*}\right) \rho(y) d y \leq C_{1} k^{\gamma+\varepsilon}, \\
\int_{\partial \mathbb{R}_{+}^{n}} w^{q}\left(y, s^{*}\right) \rho(y) d S_{y} \leq C_{2} k^{\varepsilon} .
\end{array}\right\}
$$

Step 5: Energy estimates. Let $L, \varepsilon, \delta$ and $\xi_{\ell}, \alpha_{\ell}, \omega_{\ell}, \ell=1,2, \ldots L$, be as in Lemma 6 and let $\mathcal{T}_{k}=\mathcal{T}_{k}\left(d_{k}, r_{k}, \zeta, C^{*}\right)$ be as in Lemma 3. Let $a \in \partial \mathbb{R}_{+}^{n}$ be fixed and $s^{*}=s^{*}(k, a)$ be from Step 4. Notice that (35) guarantees $\left(a^{i}, s^{*}, 0\right) \in \mathcal{T}_{k}\left(\frac{1}{2} k^{-\alpha_{\ell}}, 1, \gamma-\alpha_{\ell}+\varepsilon, C_{1} / \rho(1)\right)$ for $i=1,2, \ldots, X$ and $\ell=1,2, \ldots, L$, and (36) implies (30) with $a=a^{i}, i=1,2, \ldots, X$. Consequently, Lemma 7 implies

$$
\int_{B_{1 / 2}^{\partial}}\left(w_{k}^{a^{i}}\right)^{q+1}\left(y, s^{*}\right) d S_{y} \leq C k^{\gamma-\delta} \text { for } i=1,2, \ldots, X \text { and } k \text { large, }
$$

and using (19) we obtain

$$
\begin{aligned}
\int_{B_{R_{k}}^{\partial}}\left(w_{k}^{a}\right)^{q+1}\left(y, s^{*}\right) d S_{y} & \leq \sum_{i=1}^{X} \int_{B_{1 / 2}^{\partial}}\left(w_{k}^{a^{i}}\right)^{q+1}\left(y, s^{*}\right) d S_{y} \leq C k^{\gamma-\delta}(\log k)^{(n-1) / 2} \\
& \leq C k^{\gamma-\delta / 2} \quad \text { for } k \text { large. }
\end{aligned}
$$

In addition, since

$$
\rho(y)=e^{-|y|^{2} / 8-|y|^{2} / 8} \leq k^{-n} e^{-|y|^{2} / 8}, \quad \text { for }|y|>R_{k},
$$

we have

$$
\int_{\partial \mathbb{R}_{+}^{n} \backslash B_{R_{k}}^{\partial}}\left(w_{k}^{a}\right)^{q+1}\left(y, s^{*}\right) d S_{y} \leq C \int_{\partial \mathbb{R}_{+}^{n} \backslash B_{R_{k}}^{\partial}} k^{(q+1) \beta-n} e^{-|y|^{2} / 8} d S_{y} \leq C
$$

hence

$$
\int_{\partial \mathbb{R}_{+}^{n}}\left(w_{k}^{a}\right)^{q+1}\left(y, s^{*}\right) d S_{y} \leq C k^{\gamma-\delta / 2} \quad \text { for } k \text { large. }
$$

Denoting $w:=w_{k}^{a},(36),(9)$ and (37) imply

$$
\begin{gathered}
\left|\int_{\mathbb{R}_{+}^{n}}\left(w w_{s}\right)\left(y, s^{*}\right) \rho(y) d y\right| \leq\left(\int_{\mathbb{R}_{+}^{n}} w^{2}\left(y, s^{*}\right) \rho(y) d y\right)^{1 / 2}\left(\int_{\mathbb{R}_{+}^{n}} w_{s}^{2}\left(y, s^{*}\right) \rho(y) d y\right)^{1 / 2} \\
\leq C\left(\int_{\mathbb{R}_{+}^{n}} w^{q+1}\left(y, s^{*}\right) \rho(y) d y\right)^{1 /(q+1)} k^{\frac{\gamma+\varepsilon}{2}} \leq C k^{\frac{\gamma-\delta / 2}{q+1}+\frac{\gamma+\varepsilon}{2}} \leq C k^{\gamma-\delta / 2}
\end{gathered}
$$

provided $\varepsilon$ and $\delta$ are small enough. Finally, (6), (37) and (38) guarentee $E_{k}^{a}\left(s^{*}\right) \leq C k^{\tilde{\gamma}}$ with $\tilde{\gamma}:=\gamma-\delta / 2$ and $k$ large, and the monotonicity of $E_{k}^{a}$ implies (17). This concludes the proof. 
Remark 9. A straighforward modification of the proof of Theorem 1 provides a simpler proof of [14, Theorem 1]. One just has to replace $q$ with $p$, set $\beta:=1 /(p-1)$, replace $\mathbb{R}_{+}^{n}, \partial \mathbb{R}_{+}^{n}$ and $B_{r}^{+}, B_{r}^{\partial}$ with $\mathbb{R}^{n}$ and $B_{r}$, respectively, $(n-1)$ with $n$, and do a few more straightforward changes. In particular, (3) should be removed and (26) should be replaced with $\beta\left(\frac{p+1}{2 p}\right)^{L}<\mu$.

Remark 10. If $\beta<\mu+1$, then Lemmas 5-6 are not needed and the proof of (a modification of) Lemma 7 is simpler. In fact, the inequality $\beta<\mu+1$ implies $\gamma \frac{\mu}{\beta}>\gamma-\frac{\gamma}{\beta}$, hence if $\delta, \varepsilon>0$ are small enough and $\xi:=\gamma-\varepsilon-\delta$, then there exists $\alpha<\xi / \beta$ such that $\xi \frac{\mu}{\beta}>\gamma-\alpha+\varepsilon$. If $(a, \sigma, 0) \in \mathcal{T}_{k}\left(\frac{1}{2} k^{-\alpha}, 1, \gamma-\alpha+\varepsilon, C\right)$, then Lemma 3 guarantees $w_{k}^{a}(\cdot, \sigma) \leq k^{\xi}$ on $B_{1 / 2}^{\partial}$, hence assumption (30) implies (32) with $G_{1}:=B_{1 / 2}^{\partial}$ and $\xi_{1}:=\xi$. Consequently, (31) is true.

Notice that if $\beta=1 /(p-1)$, then the inequality $\beta<\mu+1$ is equivalent to the inequality $p<p^{*}$ in [14].

\section{REFERENCES}

[1] M. Chlebík, M. Fila: On the blow-up rate for the heat equation with a nonlinear boundary condition. Math. Methods Appl. Sci. 23 (2000), 1323-1330

[2] K. Deng, M. Fila, H.A. Levine: On critical exponents for a system of heat equations coupled in the boundary conditions. Acta Math. Univ. Comenianae 63 (1994), 169-192

[3] V.A. Galaktionov, H.A. Levine: On critical Fujita exponents for heat equations with nonlinear flux conditions on the boundary. Israel J. Math. 94 (1996), 125-146

[4] Y. Giga, R.V. Kohn: Characterizing blowup using similarity variables. Indiana Univ. Math. J. 36 (1987), 1-40

[5] J. Harada: Positive solutions to the Laplace equation with nonlinear boundary conditions on the half space. Calc. Var. 50 (2014), 399-435

[6] J. Harada: Boundary behavior of blowup solutions for a heat equation with a nonlinear boundary condition. NoDEA 27 (2020), Article 6

[7] B. Hu: Nonexistence of a positive solution of the Laplace equation with a nonlinear boundary condition. Differ. Integral Equations 7 (1994), 301-313

[8] B. Hu, H.-M. Yin: The profile near blowup time for solution of the heat equation with a nonlinear boundary condition. Trans. Amer. Math. Soc. 346 (1994), 117-135

[9] O.A. Ladyženskaja, V.A. Solonnikov, N.N. Ural'ceva: Linear and quasilinear equations of parabolic type. Amer. Math. Soc., Transl. Math. Monographs, Providence, RI 1968

[10] G.M. Lieberman: Second order parabolic differential equations. World Scientific, Singapore 2005 (revised edition)

[11] P. Poláčik, P. Quittner, Ph. Souplet: Singularity and decay estimates in superlinear problems via Liouville-type theorems. Part II: parabolic equations. Indiana Univ. Math. J. 56 (2007), 879-908

[12] P. Quittner: Liouville theorems for scaling invariant superlinear parabolic problems with gradient structure. Math. Ann. 364 (2016), 269-292

[13] P. Quittner: Liouville theorems for superlinear parabolic problems with gradient structure. J. Elliptic Parabolic Equations 6 (2020), 145-153

[14] P. Quittner: Optimal Liouville theorems for superlinear parabolic problems. Duke Math. J., to appear (Preprint arXiv:2003.13223)

[15] P. Quittner, Ph. Souplet: Parabolic Liouville-type theorems via their elliptic counterparts. Discrete Contin. Dynam. Systems, Supplement 2011 (Proceedings of the 8th AIMS International Conference on Dynamical Systems, Differential Equations and Applications, Dresden 2010), 1206-1213

[16] P. Quittner, Ph. Souplet: Superlinear parabolic problems. Blow-up, global existence and steady states. Birkhäuser Advanced Texts, Birkhäuser, Basel 2019 (2nd edition) 